\title{
Cencerradas y matracas en Navarra durante el Antiguo Régimen: funciones y objetivos
}

\author{
Javier Ruiz Astiz * \\ Archivo del Senado
}

RESUMEN: Durante el Antiguo Régimen las cencerradas actuaron en la mayoría de las ocasiones como mecanismos de control y represión de los comportamientos colectivos. En ellas sus causantes se valieron de una serie de instrumentos para realzar sus actuaciones, tratando de esta manera de legitimar sus acciones. El presente artículo tiene como finalidad estudiar estos sucesos prestando una especial atención a sus funciones y objetivos. Sin embargo, la tarea principal consistirá en profundizar en los casos vistos en el reino de Navarra, organizándolos en tres grupos: 1. enlaces matrimoniales; 2. comportamientos inmorales; 3. acciones de gobierno. Todo ello con la intención de comprender de un modo satisfactorio estas prácticas de justicia comunitaria.

\section{Palabras Clave: Cencerrada; Desorden público; Injuria; Comuni- dad; Reino de Navarra; Antiguo Régimen.}

\section{Rough Music and Skimmington in Navarre during the Old Regime: Functions and Objectives}

ABSTRACT: During the Old Regime rough musics ones acted in the majority of the occasions like control mechanisms and repression of the collective behaviors. In them their causes were worth of a series of instruments to heighten their activities, being tried this way to legitimize their actions. The present article has as an aim to study these events rendering a special attention to its functions and objectives. Nevertheless, the main task will consist of deepening in the cases seen in the kingdom of Navarre, organizing them in two groups: 1. weedings; 2. immoral behaviors; 3. gov-

* Doctor en Historia Moderna (Universidad de Navarra). 
ernment actions. All this with the intention to understand of a satisfactory way these practices of communitarian justice.

KEY WORDS: Rough music; Public disorder; Insults; Community; Kingdom of Navarre; Old Regime.

\section{INTRODUCCIÓN}

El desorden público debe ser entendido como una expresión manifiesta de la violencia popular que se ejerció durante los siglos modernos. Ésta debemos concebirla como una expresión propia que se caracterizó no sólo por tratar de alcanzar unos fines determinados, sino también por poseer un lenguaje privativo y unas tácticas específicas. Como podrá apreciarse, lo que se pretende demostrar a través de esta investigación son las distintas finalidades que adoptó un determinado tipo de altercado, como fueron las cencerradas y matracas que se originaron en el reino de Navarra entre 1512 y 1808.

Para la consecución de este artículo ha resultado primordial consultar los procesos judiciales existentes en la sección del Consejo Real en el Archivo General de Navarra, así como también los que se encuentra en la Audiencia Episcopal en el Archivo Diocesano de Pamplona. No obstante, huelga decir que este trabajo forma parte de mi proyecto de Tesis Doctoral ${ }^{1}$, en la que se han analizado un total de 336 pleitos para llegar a comprender los distintos tipos de altercados que se ocasionaron en Navarra durante el Antiguo Régimen. De dicho estudio se desprende la enorme variedad de disturbios que se originaron en Navarra durante las fechas de estudio, pudiéndose destacar de esta manera la enorme complejidad y heterogeneidad de la conflictividad que tuvo lugar.

Sin embargo, lo que a nosotros nos interesa es comprobar que las cencerradas y matracas aparecen registradas en 30 ocasiones, lo que constituye un $9 \%$ de los procesos judiciales que han sido analizados. Debido a ello, consideramos que se trata de una representación sumamente relevante como para dedicarle un estudio en profundidad. Bien es cierto que en esta ocasión la idea es centrarse en el estudio de los distintos tipos de denuncias y sanciones que se encontraron detrás de estos «rituales chariváricos» para profundizar en el transfondo de aquellos actos, sin detenernos demasiado en los aspectos formales de aquellas procesiones burlescas porque son asuntos que ya han sido ampliamente analizados en épocas pasadas por numerosos investigadores.

Por tanto, el objetivo primordial del presente artículo será mostrar la naturaleza de este tipo de manifestaciones comunitarias. Lo cierto es que toda

1 Ruiz Astiz, 2010. 
cencerrada -como señala Enríquez ${ }^{2}$ - debe ser comprendida como una expresión manifiesta de la justicia que cada comunidad vecinal ejerció durante la Edad Moderna en materia matrimonial, sexual y también en relación a la gobernabilidad local. Argumento que es corroborado también por Grinberg y Bonnain-Moerdyk ${ }^{3}$, para quienes el charivari era una manera de hacer reinar la justicia a escala local, al igual que para Usunáriz ${ }^{4}$, puesto que según éste se trataba del modo en el que cada comunidad ponía en práctica una forma de entender la moralidad colectiva. En definitiva, el objetivo final de este mecanismo cultural fue el control social ${ }^{5}$, a través del cuál manifestar, tal y como expone Shoemaker ${ }^{6}$, la desaprobación hacia determinados comportamientos. A su vez, esta práctica de control de la comunidad constituyó un ritual que se empleó para poner a prueba el honor de las víctimas ${ }^{7}$. Pudiendo morir estas últimas, socialmente hablando, a consecuencia de la humillación pública que suponía toda matraca ${ }^{8}$.

\section{ASPECTOS FORMALES}

Durante los siglos modernos la cencerrada se erigió en un juicio de carácter popular que principalmente fue empleado cuando se produjo una ruptura moral en lo que al comportamiento matrimonial o al sexual se refiere, aunque también existieron otras motivaciones. Su puesta en marcha supuso la difamación de un individuo concreto o de una pareja determinada a partir de canciones y coplillas jocosas, así como de estridentes ruidos producidos por el golpeo de cacerolas, cuernos o huesos. Fruto de aquello el clima que se respiraba durante su desarrollo estaba muy próximo a los festejos propios del Carnaval ${ }^{9}$.

Se trató, sin duda, de una protesta colectiva que mayoritariamente estuvo encabezada por la población más joven de cada localidad, aunque hubo ocasiones en que los protagonistas fueron personas mayores de 30 años. De este modo, hemos de resaltar que no se trató de un ritual que fue único y exclusivo

2 ENRÍQUEZ, 1996: 34.

3 GrinBerG, 1985: 391. BONNAIN-MOERDYK, 32/2 (Paris, 1997): 394.

4 USUNÁRIZ GARAYOA, 2006: 244.

5 BAZÁn DíAZ, 1995: 271. EIBACH, 2008: 60. MANTECÓN, 2008: 332.

6 SHOEMAKER, 1990: 208.

7 Muir, 1997: 103. Pillorget, 1975: 367. Robisheaux, 1989: 118.

8 ENRÍQUEZ, 1996: 40; THOMPSON, 27/2 (Paris 1972): 290.

9 Muir, 1997: 102. Chartier, 1986, (1) : 250; EnRíQuez, III (Bilbao, 1998): 143; PITOU, 47-1 (Paris, 2000): 77; USUNÁRIZ GARAYOA, 2006: 236; DAVIS, 1975: 121; MARGOLIN, 1975, (III) ; 600. 
de los solteros -como bien atisba Usunáriz ${ }^{10}$, aunque ello no quiere decir que el papel que detentaron los personajes casados y viudos en cada comunidad se redujese tan sólo a un consentimiento tácito según Iglesias ${ }^{11}$, o que se dedicasen a ser sus promotores como si de una mano negra se tratase como expuso Schindler ${ }^{12}$. Nada más lejos de la realidad, porque no sólo tuvieron parte activa en la configuración de dichas acciones, sino que también participaron -como sostiene Enríquez ${ }^{13}$ - de los actos burlescos e inquisitivos que se llevaban a cabo durante su desarrollo.

Como muestra de que no sólo los solteros fueron los que instigaron y tomaron parte activa en las cencerradas que se desarrollaron en la Navarra moderna nos encontramos con el caso que sucedió en la ciudad de Estella la noche del 25 de julio de 1729, en donde anduvieron cantando distintas «pullas, palabras deshonestas y poco decentes» contra algunas muchachas «diferentes mozos solteros y aún casados» ${ }^{14}$, estando entre estos últimos Andrés de Mendiola y Juan Antonio Álvarez. Algo similar ocurrió la noche del 31 de mayo de 1806 en Tafalla, en donde Francisco García y Goyena, viudo, salió a cantar «una canción como por vía de matraca o cencerrada» ${ }^{15}$ el mismo día que Joaquín de Armendáriz contrajo segundas nupcias. Junto a ello debemos recalcar, puesto que ya se ha comprobado con anterioridad, que tanto los religiosos locales como las mujeres también desempeñaron un papel relevante en dichos acontecimientos.

A su vez, otro de los aspectos característicos de estas procesiones comunitarias fue que durante su desarrollo resultó sumamente frecuente que aquellas cuadrillas se dedicasen a entonar hirientes serenatas que se encargaron de criticar determinados comportamientos de sus vecinos. Para ello no dudaron en portar todo tipo de instrumentos musicales, así como aperos de labranza y utensilios domésticos, junto a lo que cabe destacar el recurso constante al disfraz de la mayoría de sus participantes.

\section{JUSTICIA COMUNITARIA: DENUNCIAS Y SANCIONES}

La intención que persigue este estudio es mostrar estos comportamientos colectivos como un instrumento para lograr controlar de un modo eficaz la moral de una determinada comunidad. De este modo, dentro de las actitudes

10 USUNÁRIZ GARAYOA, 2006: 243.

11 IgLeSias EstePA, 19 (Santiago de Compostela, 2008): 152.

12 SCHINDLER, 1996: 339.

13 ENRÍQUeZ, 1996: 63.

14 AGN, Tribunales Reales. Procesos, núm. 205738, fol. 4.

15 AGN, Tribunales Reales. Procesos, núm. 157164, fol. 2. 
que la cencerrada se encargó de sancionar nos vamos a centrar en las tres que se han podido entresacar de la documentación consultada. En primer lugar trataremos de mostrar los casos en los que se dedicaron a injuriar a cualquier persona que contrajese matrimonio, bien porque se tratase de segundas nupcias o porque fuesen enlaces flagrantemente desiguales, e incluso porque hiciesen referencia a uniones no aceptadas por el vecindario. Tras ello, en segundo lugar, nos detendremos a estudiar los sucesos que se originaron como consecuencia de los comportamientos perniciosos de determinados personajes, entre los que cabe destacar los de índole sexual, así como también otras actuaciones inmorales como la violencia familiar, el robo o el alcoholismo. Y por último, en tercer caso, intentaremos mostrar los casos de cencerradas que deben ser entendidos como mecanismos de control político, en donde se podrán apreciar las constantes críticas que recibieron las autoridades locales con motivo de sus nefastas acciones de gobierno.

\section{Enlaces matrimoniales}

A lo largo de estos siglos todo enlace matrimonial constituyó una oportunidad excepcional para que la vecindad emitiese su veredicto llevándose a cabo lo que podríamos definir como un bautizo ritualizado para acoger en su seno a la nueva pareja. Sin duda, uno de los objetivos más clásicos y posiblemente el más estudiado por parte de los investigadores ha sido el control que ejercieron las cencerradas sobre las segundas nupcias. Como ha quedado demostrado en diversos estudios, en todo el continente europeo las prácticas chariváricas se dedicaron, en la mayoría de los casos, a sancionar la celebración del matrimonio de una persona viuda, bien fuese hombre o mujer. Durante la noche del día en el que se celebraba el enlace matrimonial los jóvenes de la localidad recorrían sus calles cantando y divirtiéndose hasta llegar al domicilio de los recién casados. Una vez llegados hasta dicho emplazamiento se dedicaban a proferir injuriosas descalificaciones no sólo contra la nueva pareja, sino también contra quienes habían intervenido en la ceremonia. El objeto final de sus serenatas, además de difamar a sus destinatarios, es que no pudiesen dormir en toda esa noche. De este modo se podría decir que la costumbre de dar matracas, aplicada al matrimonio de una persona viuda, se erigió - como señala Pitt-Rivers ${ }^{16}$ - en un mecanismo de resentimiento del orden social.

Como veremos a continuación, en Navarra durante la Edad Moderna existió la costumbre de dar cencerradas a los viudos, así como a las viudas que

16 PITT-RIVERS, 1971: 205. 
contraían matrimonio nuevamente. Dicho mecanismo socio-cultural no sólo se circunscribió a nuestro territorio, sino que son muchos los investigadores que nos han aportado sus apreciaciones desde ámbitos muy diversos ${ }^{17}$. ¿A qué pudo deberse este ensañamiento popular ante las segundas nupcias? Estamos convencidos de que los que tomaron parte en dichas actuaciones colectivas lo hicieron como si de un mecanismo regulador del mercado matrimonial se tratase. Por tanto, a través de estos comportamientos nocturnos se expresaban amonestaciones contra los enlaces desiguales, no ya sólo por la diferencia de edad de los contrayentes ${ }^{18}$, sino primordialmente porque suponía la pérdida de una nueva oportunidad de casarse para los jóvenes casaderos ante la intromisión de personas adultas en su espectro nupcial. La pérdida del control, por parte de los jóvenes, sobre lo que se ha denominado «mercado matrimonial» fue lo que provocó que éstos decidiesen tomar parte activa contra las segundas nupcias que se llevaban a cabo. Estos enlaces perturbaban el correcto funcionamiento de dicho mercado, rompiendo de este modo el equilibrio afectivo existente ${ }^{19}$.

Entre los casos encontrados en Navarra debemos destacar la preponderancia que parece ser que tuvieron las cencerradas celebradas con motivo de las segundas nupcias de viudos y viudas. Así sucedió, por ejemplo, en Burgui el 24 de julio de 1611. Sebastián Pérez, quien aquel día contrajo matrimonio con Gracia Iñiguez, viuda, sufrió la misma tarde una cencerrada en su puerta. Éste, al bajar para reprender a sus autores fue asaltado por ellos a base de pedradas, con una de las cuales fue alcanzado en la cabeza, de la que poco después falleció. Los acusados, Gregorio Camín, José Gorria, Miguel Gorria, Pascual Ibañez, Juan Mayo y Miguel Bronte, se defendieron diciendo que «no saben si ellos o quién le hubiese dado la dicha pedrada en la cabeza» ${ }^{20}$. Cabe destacar también como el 15 de agosto de 1612 en Añorbe se casó por segunda vez Miguel de Leoz. Debido a su enlace con una joven de la localidad ese mismo día un grupo de personas, entre las que se encontró el religioso local Lope de Asiain, le hicieron una cencerrada ${ }^{21}$.

Fue tan habitual que los enlaces de viudos y viudas constituyesen una oportunidad única para que los contrayentes fuesen sancionados por su comunidad, que no resulta extraño que nos encontremos con dos cuadrillas que

17 Aragonés Subero, 1986: 121. Caro Baroja, 47 (Madrid, 1980): 62. Muchembled, 1988: 27. GAUVARD y GOKALP, 29 (Paris, 1974): 696. GReER, 15/1 (Londres, 1990): 34. BELMONT, 33/3 (Paris, 1978): 654. SHERIDAN, 1993: 25. LEBRUN, 1998: 52. GÓMEZ DE VALENZuela, 3 (Huesca, 1987): 120. Burguière, 1981: 45. SEgalen, 1981: 73. BerCÉ, 1974, (1): 215. KlAPISCH-ZuBER, 1985: 264. DAVIS, 50 (Oxford, 1971): 65. INGRAM, 1988: 177.

18 RUFF, 2001: 162. SCHINDLER, 2002: 155. DAVIS, 1975: 105.

19 Nicolas, 2002: 449. BurguiÈre, 1979: 92. DeAn, 2007: 123. Davis, 1975: 106.

20 AGN, Tribunales Reales. Procesos, núm. 2240, fol. 5.

21 ADP, Audiencia Episcopal. Procesos, C/ $475-\mathrm{n}^{\circ} 12$. 
salieron de noche a dar matracas al mismo matrimonio. En numerosas ocasiones el encuentro callejero de dichas pandillas trajo consigo enconadas disputas armadas fruto del acaloramiento y la altanería de sus participantes. Francisco Sainz, el 3 de octubre de 1660 en Sesma, anduvo entre las ocho y nueve de la noche «con ocasión de hacer una cencerrada, holgándose en compañía de otros mozos, que eran Diego Solano, José Solano, José Román, Juan de Pinillos y Gregorio Sanz». Esta cuadrilla, a lo que iba hacia la casa de María Solano, viuda, quien había contraído segundas nupcias aquel día, observó cómo eran atacados por Jerónimo Solano, Luis Pérez y Francisco Romeo. Como consecuencia de aquella pendencia cayó herido de un puntazo «en el pecho, en mitad de él»»22 Francisco Sainz.

Es de interés también el suceso que tuvo lugar la noche del día de Pascua de Resurrección de 1694 en la localidad de Yelz. Allí se produjo una cencerrada con motivo del matrimonio que se había celebrado entre Mariana de Urroz, ama de Martín de Izco, abad del lugar, y Francisco Baraciarte, molinero viudo. Sus «verdugos», Pedro Jaureguiberri, Miguel de Iriso y Juan López «dieron cierta cencerrada o matraca causando con ella mucha nota e inquietud», además «con descompostura maltrataron gravemente a Don Martín de Izco» ${ }^{23}$. El 3 de febrero de 1733 en Tafalla, «a lo que serían las diez horas y media» ${ }^{24}$, el objetivo de Gabriel Martínez, Martín del Boiro, José de Aguirre y Francisco de Abinzano era dar una matraca a la hija viuda de Martín de Zemborain. Finalmente fueron reprendidos y se logró evitar tal perturbación del orden público. No sucedió igual en la localidad de Yanci cuando el 4 de febrero de 1770 se produjo cierta cencerrada y alboroto nocturno. Gabriel de Maestruarena, José Antonio de Irisarri y Martín José de Samper, entre otros, anduvieron apedreando la casa de Errandonea hasta que se disolvieron cuando llegaron las autoridades locales a impedirles que continuasen con su actitud. En Tafalla, el 31 de mayo de 1806 Joaquín de Armendáriz, con motivo de sus segundas nupcias, fue objeto de una matraca. Francisco García Goyena fue su autor, quien le cantó mientras corría a caballo por las proximidades del río. Según Manuel de Iribas, testigo, entonó la siguiente canción en varias ocasiones:

\footnotetext{
Un cura y un escribano

han hecho este casamiento.

Sea en todo muy feliz

por muchos años sin cuento.

Armendáriz con paciencia
}

22 AGN, Tribunales Reales. Procesos, núm. 76051, fol. 19.

23 AGN, Tribunales Reales. Procesos, núm. 204748, fol. 1.

24 AGN, Tribunales Reales. Procesos núm. 126570, fol. 10. 
hoy segunda vez se casa, y el casamiento que ha hecho ha llevado ruido en Tafalla ${ }^{25}$.

Al margen de las cencerradas que ocasionaron las segundas nupcias, en numerosas ocasiones se produjeron también actos chariváricos con ocasión de enlaces matrimoniales que no eran aceptados por los miembros de una determinada localidad por muy diversos motivos. Entre estos últimos, cabe destacar los ataques que se profirieron contra personajes foráneos que irrumpían en el mercado matrimonial local haciendo disminuir las posibilidades de casarse de los jóvenes de dichas poblaciones ${ }^{26}$. Un ejemplo muy significativo de estas circunstancias nos lo encontramos en julio de 1570 en la localidad de Olite. Allí el origen castellano de Jerónimo de Carrión, quien se había casado con María de Estrada, provocó que «algunos vecinos de la dicha villa con ánimo y propósito diabólico, y de injuriar y afrentar» ${ }^{27}$ se dirigiesen a la casa donde se hallaban los recién casados. Frente a su domicilio comenzaron a entonar distintos versos en los que le acusaban de diversas circunstancias de la siguiente manera:

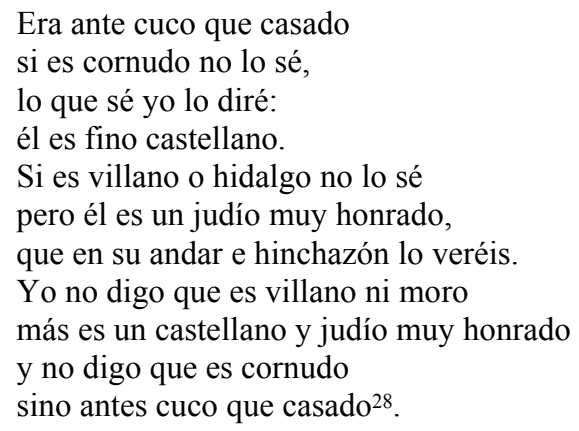

Pese a ello, también resulta imprescindible referirse a la oposición sistemática que se experimentó en otros casos por parte de los jóvenes casaderos como consecuencia de la pérdida de su amada. Este tipo de resentimiento no fue único y exclusivo de Navarra, pues se trató de un fenómeno extendido por todo el continente europeo ${ }^{29}$. Merece la pena resaltar uno de estos sucesos, ya que Pedro González y María Fidalgo contrajeron matrimonio el 8 de enero de

25 AGN, Tribunales Reales. Procesos, núm. 157164, fol. 7.

26 Muchembled, 1994: 48. Gauvard, 1982: 240. Muir, 1997: 99.

27 AGN, Tribunales Reales. Procesos, núm. 38206, fol. 138.

28 AGN, Tribunales Reales. Procesos, núm. 38206, fol. 138.

29 Pellegrin, 1983: 252. Burguière, 33/3 (Paris, 1978): 647. Marcel-Dubois, 1975: 614. 
1589 en Miranda de Arga. El mismo día del casamiento, según Catalina Remírez, «entre las doce y la una de la media noche oyó que estaban dando música con un rabel o vigüela de $\operatorname{arco»}{ }^{30}$. Entre las personas que participaron en esta matraca -Diego Bueno, Domingo Bueno, Jorge Bueno, Pascual Musgo, Pedro Muez y Juan de Gadea- se encontraba un ferviente admirador profundamente enamorado de la recién casada. María Tapiz se preguntaba «¿cómo se ha casado Pedro González con María Fidalgo, andando enamorado con ella Jorge Bueno?» ${ }^{31}$. No contentos con ello, más adelante, la noche del 24 de enero volvieron a dar voces desafiando a Pedro González, pues según los demandantes, «se va jactando que aunque sea más casada la dicha María Fidalgo con el dicho Pedro González le ha de dar músicas y seguirla» ${ }^{32}$. En otros casos parece que la costumbre era que toda pareja recién casada recibiese la bendición de la comunidad en forma de jocosa cencerrada. Así la noche del 2 de octubre de 1717 en Sesma fueron Juan Antonio Solano, Jerónimo Ruiz, Pedro Ganuza, Martín de la Fuente y Lucas Romero frente a la casa de José López García con motivo de la boda de éste con Francisca Mangado ${ }^{33}$. Un suceso similar tuvo lugar en la localidad de Fustiñana en 1703, puesto que allí se celebró una matraca para sancionar la nueva unión entre Martín de Salvatierra y Magdalena de Sola $^{34}$.

Una vez analizadas las cencerradas que se originaron con motivo de los enlaces matrimoniales, cabe destacar que en la Navarra moderna la causa primordial de estos acontecimientos fueron las segundas nupcias frente a las uniones que no eran aceptadas por la comunidad por tratarse de personajes foráneos o por no satisfacer las demandas de sus enamorados. Algo similar sucedió en el resto de Europa, destacando la preponderancia de las matracas en las que los destinatarios eran viudos o viudas ${ }^{35}$. Sin embargo, lo más destacado es que a lo largo de estas líneas se ha puesto de manifiesto que este tipo de prácticas -como señalan Bercé ${ }^{36}$ y Schindler ${ }^{37}$ - constituyeron un instrumento ejemplar para la defensa y la protección de la moral y el honor comunitario.

30 AGN, Tribunales Reales. Procesos, núm. 213107, fol. 16.

31 AGN, Tribunales Reales. Procesos, núm. 213107, fol. 17.

32 AGN, Tribunales Reales. Procesos, núm. 213107, fol. 15.

33 ADP, Audiencia Episcopal. Procesos, C/ $1755-\mathrm{n}^{\circ} 9$.

34 AGN, Tribunales Reales. Procesos, núm. 216642.

35 ThOMPSON, 2000: 550. GILlis, 1968: 32.

36 BERCÉ, 1976: 40.

37 SCHINDLER, 1996: 315. 


\section{Actitudes inmorales}

A lo largo de estas líneas el objetivo es demostrar cómo las cencerradas actuaron en múltiples ocasiones como mecanismos de control de los comportamientos colectivos. Por encima del resto, las actitudes calificadas de inmorales fueron el centro de las críticas y reprobaciones comunitarias. No cabe duda de que la gravedad de las injurias, las canciones o las coplas que fueron entonadas se vio acentuada por la presencia de testigos. Ello propició la divulgación de las difamaciones a modo de rumores ${ }^{38}$, lo que muchas veces impedía reparar los daños infringidos en la fama de las personas denostadas ${ }^{39}$. Pero, ¿quiénes fueron sus blancos preferidos? A simple vista, aunque los hombres también sucumbieron ante estos ataques -encarnados preferentemente en la figura de los religiosos locales-, fueron las presuntas actitudes lujuriosas de las mujeres las que centraron sus ofensas. No importaba su estado, bien fuesen solteras, casadas o viudas, lo cierto es que su honor resultó mancillado constantemente a través de las ofensas verbales ${ }^{40}$.

Entre sus participantes nos encontramos de nuevo a los más jóvenes de cada localidad, aunque gracias al apoyo y consentimiento de los adultos lograron conferir a sus acciones el estigma social y cultural necesario para perturbar el devenir de sus víctimas. A través de estas cencerradas lo que sus autores lograron fue poner de manifiesto ante toda la vecindad determinadas actitudes y ciertos comportamientos que algunos personajes habían llevado a cabo, y que no estaban acordes con el sistema de valores existente. Uno de los ataques más generalizados a lo largo de estas centurias fueron las acusaciones de adulterio, aunque tampoco hemos de olvidarnos de las constantes denuncias de amancebamientos, así como las imputaciones de actitudes lascivas y lujuriosas tanto de hombres como de mujeres. No obstante, en dichos actos de reprobación colectiva también se atacó al marido consentidor de la infidelidad de su mujer, al igual que tampoco se olvidaron de sancionar los malos tratos y las agresiones que se producían en el seno matrimonial, así como también otro tipo de conductas consideradas como perjudiciales (caso del alcoholismo, el robo, la falsedad y la avaricia).

En definitiva, lo que se pretendía a través de estas actuaciones era intentar redimir a toda una localidad a la vez que se castigaba a los que habían ofendido el bienestar espiritual de su comunidad. Por tanto, estas ceremonias populares deben ser comprendidas como un instrumento de crítica contra todos aquellos que habían transgredido las normas de convivencia ${ }^{41}$. Una de las

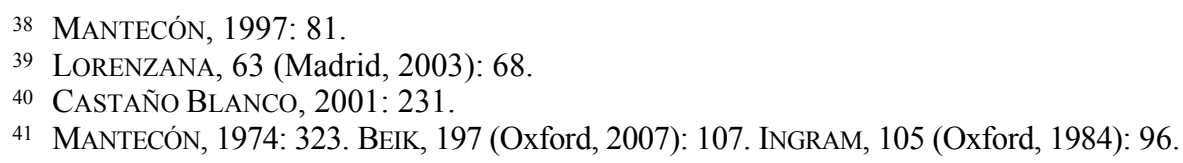


principales causas que provocaron que dichos sucesos fuesen tan generalizados durante estos siglos fue que las noticias y los chismes relativos a las vidas ajenas circularon con suma fluidez. No debe extrañarnos, como apunta Mendoza Garrido ${ }^{42}$, que muchos habitantes tuviesen conocimiento de los comportamientos inmorales de sus vecinos.

Dentro de las actitudes inmorales que fueron sancionadas cabe referirse, en primer lugar, a las descalificaciones y las mofas que se lanzaron contra las mujeres. En lo que a ellas hace referencia nos encontraremos ataques contra cualquier tipo de estado civil, bien fuesen éstas solteras, casadas o viudas. Lo cierto es que ninguna se libró de ser objeto de sus hirientes acusaciones. Respecto a las primeras hemos de indicar que su principal virtud era la conservación de su virginidad, ahí radicaba el honor de las mujeres solteras. De este modo, la pérdida de aquélla fue el motivo principal de los ataques de los que fueron objeto ${ }^{43}$. Dentro de los casos analizados podemos apreciar el que tuvo lugar en la ciudad de Estella el 25 de julio de 1729. Aquella noche se cantaron en la calle la Rúa, diferentes chanzas y palabras descompuestas contra las hijas solteras de Antonio de Cartagena, Micaela y Manuela, «tratándolas con diferentes injurias a sus estados». Los acusados, Andrés de Mendiola, José de Villanueva, Juan Antonio Guillén y Juan Antonio Álvarez, anduvieron cantando mucho rato, y entre otras cosas dijeron, aunque no se sabe a cuál de ellas: «va por los Llanos, le dan dinero porque tiene la chica dos agujeros» ${ }^{44}$. Igual sucedió el 10 de noviembre de 1744 en la ciudad de Pamplona, en donde a cosa de las doce horas de la noche hubo una cencerrada protagonizada por Nicolás Salinas, Lucas de Oronoz, José de Sada, Agustín de Lazcano y Francisco Chaparro, presbíteros, contra las hijas de Tomás de Sarasa. Éstos «con mucha gritería y tañendo una guitarra» en la calle Mayor hicieron burla y mofa de Micaela, y al mismo tiempo cantaron contra su hermana, Lorenza, de la siguiente manera:

¡Ay Lorenza, hija del sillero!, que te has dejado de engañar de un hijo de Villava, anda pensamiento, y dile a Gorraiz que no se olvide.

¡Ay hija del sillero!, no tienes que bajar a escribir sobre el tablajero porque si pasa Gorraiz [...].

¡Oyes!, mira que no ha estudiado o no sabe filosofía, cuando te pones a escribir en el tablero cartas $[\ldots]$.

No te pongas a escribir encima del tablero, que se te abrirá el agujero y te lo tapará luego. El estudiante que sabes, sabe poca filosofía, porque siempre piensa en $\mathrm{ti}^{45}$.

42 Mendoza Garrido, 1999: 269.

43 SCHINDLER, 1996: 319. MuCHEMBLED, 1994: 49. RufF, 2001: 162. KLAPISCH-ZuBER, 1985: 277. ENRÍQUEZ, 1999: 223. MANTECÓN, 1997: 73.

44 AGN, Tribunales Reales. Procesos, núm. 205738, fol. 4.

45 ADP, Audiencia Episcopal. Procesos, C/ $1603-\mathrm{n}^{\circ}$ 6, fol. 2. 
¿Qué ocurrió cuando las mujeres vejadas estaban casadas? ¿Qué tipo de descalificaciones recibieron? Ya de casada lo más importante fue el mantenimiento de la fidelidad y el bienestar del marido. Pese a ello, resultó habitual la acusación de adulterio contra muchas mujeres. Estas imputaciones no sólo deshonraban a ellas mismas, sino también a sus familiares, dentro de los cuáles sus esposos fueron los más perjudicados. Sin duda, el adulterio femenino - como indica Enríquez ${ }^{46}$ - marcaba al marido que no había sido capaz de modificar el comportamiento deshonroso de su mujer. La infidelidad de ésta constituía su defunción social, pero no la de su marido, aunque como sostiene Pitt-Rivers ${ }^{47}$ suponía un claro desafío a la hombría de éste. Pese a ello, también existieron casos en los que el hombre fue el destinatario de dichos ataques por permitir las prácticas sexuales de su esposa, de las cuáles tenía perfecto conocimiento, esto es lo que Pérez Teijón ${ }^{48}$ define como el marido consentidor. Lo cierto es que durante los siglos modernos las procesiones nocturnas se emplearon constantemente contra la infidelidad conyugal. Varias faltas sexuales podían ocasionar la celebración de cencerradas. Uno de los motivos más frecuentes fue el adulterio entre personas casadas ${ }^{49}$.

En el caso navarro debemos prestar atención al ruido y alboroto que se ocasionó el 25 de diciembre de 1595 en la villa de Sangüesa. Allí podemos comprobar las acusaciones que se vertieron en las canciones que entonaron Pedro de Orbaiz, Guillén de Grez, Salvador Zurita, Juan de Ansó y Martín de Rocaforte. Esta cuadrilla, entre once y doce horas de la noche, arremetió contra varias vecinas de la localidad. De este modo, según Vicente Nápoles, a Lorenzo Castillo le gritaron:

¡Hola bellaco!, yerno de Villalobos, envía la mujer de Pedro Gómez, que él la está esperando en el barrio de Mediavilla y envía a tu mujer a Martín, el carnicero, que él le dará buena carne ${ }^{50}$.

Los acusados siguieron con su perorata, y como relata Graciana de Aldabe, a Juan Navarro le dijeron:

¡Hola Santo de Orta!, que vos por cuatro reales que dejas después hacer de tu mujer lo que quieren ${ }^{51}$.

46 ENRÍQUEZ, 1996: 218.

47 PITT-RIVERS, 1971: 142.

48 PÉREZ TEIJÓN, 1995: 143.

49 Gauvard, 1982: 241. Morgan; Rushton, 2007: 117. Medick, 1988: 323. AmusSen, 1995: 59. CASTAÑO BLANCO, 2001: 230.

50 AGN, Tribunales Reales. Procesos, núm. 99628, fol. 2.

51 AGN, Tribunales Reales. Procesos, núm. 99628, fol. 3. 
Graciana de Azpilcueta sostiene que Pascual de Garayo también fue objeto de las difamaciones y acusaciones, cantándole:

¡Hola Pascualico Gascón!, que te has casado con la amiga de Martinete y la has tomado agujereada y por el celaje te han dado treinta ducados ${ }^{52}$.

No conviene olvidar que una de las manifestaciones más típicas durante estos actos fue la colocación de cuernos en las puertas de las casas de los maridos atacados, así como el empleo de la expresión cornudo para referirse a ellos en sus burlonas serenatas. De este modo, puede apreciarse cómo en la localidad de Estella en 1565 fueron hallados en la puerta de Diego Navarro «dos cuernos a los lados» ${ }^{53}$. Dicha acción no resulta nada novedosa porque algo similar tuvo lugar en Marcilla en 1617, en donde se encontraron unas «sartas de cuernos y otras cosas colgadas en las puertas» ${ }^{54}$.

Por otro lado, ni siquiera las viudas se libraron de ser objeto de los fervientes ataques encarnados en la sanción de ciertos comportamientos sexuales. Al igual que sucedió con solteras y casadas, también las mujeres que habían enviudado quedaron expuestas al oprobio y a la crítica moralizante ${ }^{55}$. Así sucedió, por ejemplo, en la ciudad de Tudela a principios de junio de 1609. Allí fueron hallados dos libelos difamatorios dirigidos contra Ángela de Egea, donde era acusada repetidas veces de amancebamiento, diciendo de ella, en el primero «quien quisiere hacer tal cosa acuda a la viuda de Jubera», y en el segundo «no se espante la Jubera, pues clérigos y frailes tratan con ella» ${ }^{56}$. Como puede apreciarse, en ocasiones los escritos infamantes se erigieron en una herramienta más para la crítica ${ }^{57}$.

Pero no todo quedó ahí, el control de ciertas actitudes en algunos casos fue más lejos que la mera crítica ante ciertos comportamientos sexuales ilícitos, sino que también publicitaron la actitud bárbara que, dentro de la vida conyugal, tenían algunos maridos con sus esposas. Cabe destacar las condenas populares contra la violencia marital. A lo largo de la Edad Moderna los vecinos de muchas localidades fueron espectadores que tomaron parte activa en la sanción y denuncia de dichos comportamientos nocivos en el seno matrimonial ${ }^{58}$. De este modo, cuando los malos tratos de los que eran víctimas las mujeres eran patentes para el resto de la comunidad no dudaron en reproducir

52 AGN, Tribunales Reales. Procesos, núm. 99628, fol. 4.

53 AGN, Tribunales Reales. Procesos, núm. 67200, fol. 42.

54 AGN, Tribunales Reales. Procesos, núm. 89535, fol. 1.

55 UNDERDOWN, 1985: 101. INGRAM, 1988: 171. WALTER, 2008: 110.

56 AGN, Tribunales Reales. Procesos, núm. 100653, fol. 64.

57 Véase RuIz Astiz, 2012: 103.

58 RuIZ Astiz, 2013. 
constantes cencerradas alertando de sus perniciosas actitudes ${ }^{59}$. No debe sorprender, por tanto, que en la cencerrada que se celebró en diciembre de 1595 en la localidad de Sangüesa, Graciana Pérez nos informe sobre las acusaciones de malos tratos que sus participantes dirigieron a Pedro de Ustés de la siguiente manera:

¡Hola Perico de Ustés!, el que aporrea y dá de palos a su mujer cuando está borracho, véte a casa de Abadía ${ }^{60}$.

Detrás de otros sucesos, como ya he indicado con anterioridad, se encontró la dura reprobación de la comunidad ante ciertas prácticas perniciosas para el buen orden de la institución familiar. Dentro de estas últimas cabe destacar las acusaciones de un excesivo consumo de alcohol, así como las difamaciones dirigidas contra el honor familiar. No debe sorprendernos, por ejemplo, el «grande ruido y escándalo» ${ }^{61}$ del que fueron objeto Juan de Azcárate, y su esposa, Catalina del Pueyo, vecinos de Tafalla. El 26 de enero de 1630, Domingo de Sola, Carlos de Berruezo y Luis de Berico, entre las ocho y nueve horas de la noche, «empezaron a darle matraca y decirle palabras de pesadumbre mudada la voz» ${ }^{62}$, tratándolo de «borracho, borrasquilla pudiente, bellaco y otras palabras ofensivas ${ }^{63}$. Similar fue el caso que tuvo lugar en Pamplona el 26 de abril de 1613. Allí, a lo largo de la calle Zapatería, Jerónimo de Errazquin, Juan Sanz de Aguilar, Juan García y Antonio Gutiérrez, fueron «dando voces y diciendo contra algunas personas honradas de la dicha calle muchas palabras injuriosas nombrando por sus nombres a tales personas ${ }^{64}$, todo lo cuál parece que estuvo escrito en «tres pliegos de papel» en donde se recogieron «ciertas coplas e injurias» ${ }^{65}$ contra el honor de sus familias.

Por último, no debemos olvidar las acusaciones que se profirieron contra algunos miembros del estamento clerical. Estas críticas fueron relativamente abundantes durante el desarrollo de prácticas chariváricas en la Navarra de los siglos modernos. Ello es buena muestra del celo con el que la comunidad aguardó y protegió a sus miembros, así como la connivencia con las normas y directrices que concilios y sínodos habían puesto en marcha para desterrar comportamientos inmorales entre los miembros de la clerecía. Y una de las

59 FOYSTER, 2005: 196. MANTECÓN, 2002: 27; BEDNARSKI, 2003: 295. DOBASH, 28/5 (Berkeley, 1981): 565. ShOEMAKer, 26/2 (Londres, 2001): 206. CASHMERE, 21/3 (Londres, 1991): 307.

60 AGN, Tribunales Reales. Procesos, núm. 99628, fol. 3.

61 AGN, Tribunales Reales. Procesos, núm. 74255, fol. 2.

62 AGN, Tribunales Reales. Procesos, núm. 74255, fol. 4.

63 AGN, Tribunales Reales. Procesos, núm. 74255, fol. 8.

64 AGN, Tribunales Reales. Procesos, núm. 297228, fol. 2.

65 AGN, Tribunales Reales. Procesos, núm. 297228, fol. 3. 
formas con las que contamos para poder apreciar el éxito que alcanzó fueron las cencerradas de corte moral que se sucedieron en el espacio y en el tiempo en toda Europa ${ }^{66}$. Así parece que sucedió uno de estos casos en la localidad de Cascante. El 23 de diciembre de 1566 fueron hallados unos carteles y libelos difamatorios contra la honra y fama de Pedro Garcés ${ }^{67}$. Detrás de estos escritos se encontraron personajes de la localidad como Rodrigo Rebolledo, Miguel López, Miguel Garcés, Domingo Camuel, el Licenciado Sánchez, Pedro de Orta, Pedro Jiménez, Pedro Ruiz, Francisco Jiménez, Melchor Sánchez, Melchor de Esparza, Diego de Ágreda, Catalina del Bayo y Francisco de Arguedas. Los ataques que en aquellas proclamas se contenían eran los siguientes:

Don Pedro Garcés, hombre malo y maldito engendrado en pecado y en maldición, y contra ley divina, hijo de padres malditos, pésimos y malvados. Di bestia bruta sensual, ¿hasta cuándo has de perseverar en tus maldades? Mira que por los pecados tuyos y por los pecados de aquellos que contigo andan tiene Dios olvidado a este pueblo de Cascante, y está airado contra el disodomita excomulgado, bestia bruta, ¿con que ánimo te pones delante el pueblo todos los días a decir misa y a tratar los sacramentos, durmiendo todas las noches con tus putas y amigas? La virtud y bondad de este pueblo tú la has desterrado y has sembrado en él a Sodoma y a Jerusalén. Por tus pecados padecemos ya muchos años, hambres y persecuciones. Tú y los ladrones de tu casa nos robáis, el pueblo, capos, viñas y güertas, frutas y hortalizas, y todo es para tus putas. Mira que tus maldades están públicas y manifiestas por todo el mundo. Ahora dos años cuando andaban robando en la güerta de Tudela, en todo el Reino se dijo públicamente que eran los ladrones Alquenicuo Zarain y Sancho Garcés y que tú los acogías en tu casa. Mira cuantas mujeres has deshonrado. A la mujer de Cervantes te tuviste tres o quoatro años por amiga y la dejaste por pobre, ahora que ha heredado ya haste tornado a adulterar y a encarnizarte con ellas, otra puta tienes en casa para cada noche y después vas a decir misa al pueblo. ¡Oh luterano traidor! No sé como Dios disimula contigo. Tus rentas y señoríos te han de ser quitadas, y si esto no quisiere hacer el Obispo, el pueblo irá contra ti y como leones hambrientos devorarán y espedazarán a tí y a tus ladrones ${ }^{68}$.

Según se recoge en la queja criminal que presentaron los injuriados «muchos de los acusados o los más de ellos leyeron los dichos libelos y los anduvieron leyendo y mostrando a todos», y peor aún «sin haberlos quemado ni rasgado», logrando que todo el vecindario «tuviera noticias de ellos» ${ }^{69}$. Igualmente, la noche del 26 de agosto de 1612 en la localidad de Arre andu-

66 Candau Chacón, 1993: 256. Stevenson, 1992: 59. Slack, 1984: 90. Krausman BEN-AMOS, 1994: 203.

67 Véase Ruiz Astiz, 12 (Pamplona, 2008): 20-21.

68 AGN, Tribunales Reales. Procesos, núm. 97540, fol. 50.

69 AGN, Tribunales Reales. Procesos, núm. 97540, fol. 223. 
vieron varios vecinos por las calles «con cencerros, campanas, flautas y con cuarteles $\gg{ }^{70}$. Martín de Echarri, Juan de Artica, Juan de Araiz, Lope de Iribas, Juan de Iribas, Juan Sanchotena, Juan de Labiano, Bernardo de Oricain y Miguel de Sorauren, anduvieron «haciendo ruido y música» ${ }^{71}$. El objeto de sus pullas fue el vicario Jerónimo de Berrio, pues su comportamiento era más que dudoso. De este modo, frente a su casa fue tachado de «garroso y harto de piernas, y que era pobre», dando a entender en sus cantares que «anda tras de sus enamoradas», cantándole en euskera:

\author{
Arza doaye lasterrean \\ gauza duela viz[c]arrean, \\ orra! Don Xeronimorec \\ badu [bondi] bat çorri vizcarrean ${ }^{72}$. \\ Erbia doaye lasterrean \\ conexua darrayola açean \\ orra! Don Hieronimoric \\ bere amoranten açean $^{73}$. \\ Orra Don Hieronimo! \\ bere auçia, galduric \\ Çilbeti Arretera mandate \\ errumes caminoam barrena \\ don Jeronimo ouena ${ }^{74}$.
}

Los vecinos de Arre que protagonizaron estas durísimas acusaciones se dirigieron también contras las mujeres casadas de las casas de Galantena, Echarlesarena y Alamán. En la primera de ellas cantaron una coplilla en la que decían que «el vicario había tomado dineros a censo, siendo fiador de Martín de Saragüeta, dueño de la dicha casa y que su mujer pagaba los censos». En la puerta de Echarlesarena entonaron la siguiente copla:

$$
\begin{aligned}
& \text { Mari Martinori tristeric, } \\
& \text { nola ezpayto miraric } \\
& \text { don Jheronimori xarriçayo } \\
& \text { dolu mauntua jançiric. } \\
& \text { Aniçetan joanbazayo } \\
& \text { gueçuran, diotic }
\end{aligned}
$$

70 AGN, Tribunales Reales. Procesos, núm. 41424, fol. 17.

71 AGN, Tribunales Reales. Procesos, núm. 41424, fol. 17.

72 "El oso va corriendo / con una cosa en la espalda, / ¡Orra! Don Jerónimo / tiene una gran pulga en la espalda».

73 "La liebre va corriendo / mientras le sigue el conejo / ¡Orra! Don Jerónimo / detrás de sus amantes».

74 “¡Orra Don Jerónimo!, / su juicio perdido / lo han llevado de Cilbeti a Arre / por el camino de los peregrinos / Don Jerónimo pecador». 
erratera ollasco

parea $\operatorname{arturic}^{75}$.

Antes de llegar a la última de las casas mencionadas, y en la puerta de la Casa de Ezquerrexena, nombraron a la dueña de aquella que era viuda y le cantaron al vicario, nuevamente, las coplas anteriormente descritas. Una vez en la casa Alamán, su dueña fue injuriada y calificada como «puerca sucia», y a su esposo, Juan de Artica, «lo trataron de falso», aunque lo peor recayó sobre Antona Alamán, a quien le entonaron lo siguiente:

Don Jheronimori tristeric
nola expaytu miraric
Antona Alamanecori,
joan çayo consolaçera galantic ${ }^{76}$.

Mientras, en otras puertas decían «nombrando a las dueñas, que fuesen al dicho vicario con pollos y garrafas de vino frío» $\rangle^{77}$. Parece ser que, poco después de que los acusados fuesen puestos en libertad bajo fianza, según nos relata Juana de Echalar, la «víspera de Nuestra Señora de septiembre y el día de San Miguel de septiembre», a la noche, «dieron muy grande cencerrada». El 8 de septiembre, después de anochecer, «salieron con mucho ruido y alboroto con muchos cencerros y espadas desenvainadas, tirando muchas cuchilladas y sacando centellas», «tirando muchas pedradas a los tejados y ventanas», y lo mismo hicieron la noche del 29 de septiembre. Lo más destacado es que, según la testigo, «no hubo cencerrada ni otras cosa ninguna mientras estuvieron presos los acusados» ${ }^{78}$.

Un caso similar acaeció en Ibero la noche del 23 de agosto de 1733. Allí, entre las nueve y diez horas, llegaron a la puerta de la casa del vicario de la localidad diferentes sujetos con un juglar, y anduvieron danzando y cantándole durante un buen rato. Dirigiéndose debajo de la ventana de la habitación donde dormía este personaje comenzaron a cantarle:

Padre cura, a vuestra merced que lo entiende, alargue la mano, compóngame el dengue.

75 «A la triste María Martino, / cómo no es de maravillarse / se ha acercado a Don Jerónimo / vestida con un manto de duelo / Si a menudo le ha ido / diciendo falsamente / que llevaba / un par de gallinas».

76 «Al triste Don Jerónimo / cómo no es de maravillarse / a Antona Alamán, / le ha ido a consolar galantemente».

77 AGN, Tribunales Reales. Procesos, núm. 41424, fol. 18.

78 AGN, Tribunales Reales. Procesos, núm. 41424, fol. 71.

Hispania, 2013, vol. LXXIII, nº. 245, sept.-diciembre, págs. 733-760, ISSN: 0018-2141, e-ISSN: 1988-8368, doi: 10.3989/hispania.2013.020 


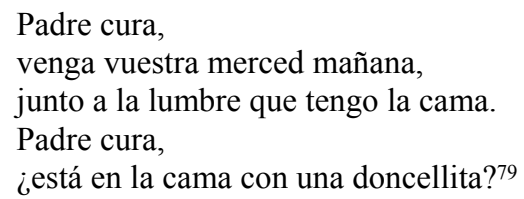

A lo que entonces salió a la ventana dicho vicario y les dijo: «caballeros, cesen de cantar y déjenme descansar». Lo que motivó que empezasen a tirar pedradas con mucho ímpetu contra su ventana ante el grito de «justicia, justicia» del ofendido. Entre los participantes en esta cencerrada contra la supuesta inmoralidad del párroco de Ibero se encontraron Juan Martín de Urdaniz, Martín de Erro, Pedro Juan de Iralzu y Agustín de Ballarian. Y pese a las acusaciones por partes de éstos de mantener tratos ilícitos con una joven, parece ser que las verdaderas motivaciones fueron otras muy distintas. El origen de la animadversión que sentían hacia este personaje vendría desde dos años antes, «con el motivo de la oposición que hacía a las danzas» ${ }^{80}$.

Para concluir, a modo de recapitulación, podemos advertir que ha quedado puesto de manifiesto que numerosas cencerradas se celebraron -como sostiene Pitt-Rivers ${ }^{81}$ - cuando las normas morales habían sido puestas en entredicho. Sus transgresores se convirtieron en sus principales víctimas, pudiendo pagar la pérdida de su honra -según Caro Baroja ${ }^{82}$ - con la pérdida de la vida. Aunque ya hemos comprobado que en algunos casos los mayores afectados, caso de los maridos y demás familiares, lo fueron también de un modo indirecto.

\section{LA CENCERRADA ENTENDIDA COMO CRÍTICA POLÍTICA}

Si durante los siglos modernos hubo un método hábil y despiadado de crítica de carácter político ese fue la cencerrada, puesto que ésta también adoptó un ensañamiento mordaz con determinadas personalidades a escala comunitaria. Debido a ello, a continuación es nuestra intención analizar este mecanismo como un instrumento de control político ejercido en diversas localidades. Dentro de las actitudes que esta variante de la cencerrada se dedicó a señalar nos centraremos preferentemente en las acusaciones emitidas con motivo de la forma de ejercer la autoridad, así como en las jocosas descalificaciones que se profirieron contra dichos personajes no ya sólo por tratarse de personajes foráneos, sino también por ser fruto de odios y rencores anteriores.

\footnotetext{
79 AGN, Tribunales Reales. Procesos, núm. 242235, fol. 2.

80 AGN, Tribunales Reales. Procesos, núm. 242235, fol. 3.

81 PITT-RIVERS, 1971: 203.

82 Caro Baroja, 1968: 81.
} 
Sin duda, la enemistad y el odio lastrado con el paso de las generaciones hacia los elementos descendientes de familias judaizantes provocó la aparición de libelos y pasquines en donde se lanzaban durísimas acusaciones contra ciertos individuos sobre sus orígenes y sus prácticas religiosas. No debe extrañarnos, por tanto, que nos encontremos algún caso en el que una cencerrada fue empleada para manifestar de modo mucho más abierto y público el rencor que sentían hacia familias de sangre judía. De este modo, a finales de julio de 1592 en Corella, Miguel de Rivas y su hermano, Pedro de Rivas, fueron objeto de una serie de ataques públicos. Según nos relata Diego Fernández, testigo que vivía junto a la casa de los injuriados, «sintió gran ruido de gente andando», los cuales iban «dando voces y tirando pedradas» ${ }^{83}$. Los culpables fueron Domingo Virto, Juan Virto, Pedro Vicente, Ambrosio Escudero y Domingo Maza, todos vecinos de dicha localidad. Todos ellos le gritaban a voces «vuélvete, vuélvete judío negrillo, nos quieres apedrear, no basta que apedreaste a Cristo, y tú, regular, que si no fuese por las órdenes que tienes te haríamos que la mayor pieza de tu persona fuese la oreja» ${ }^{84}$. Estas palabras y otras semejantes les dijeron desde la calle, «duplicándolas por muchas veces y cantando, riendo y con mucho menosprecio» ${ }^{85}$. Marco Muro, testigo presencial, nos relata las injuriosas frases que les dedicaron a los hermanos Rivas, diciéndoles por muchas veces: "salid aquí bellacos, perros, judíos quemados en sambenitados, que voto a dios os hemos de abrasar en vuestra propia casa y agradeced, vos y regular a las órdenes que tenéis, que de otra manera os habíamos de quemar dentro de casa» ${ }^{86}$. Por último, según Francisco Fernández, los demandados les dijeron a grandes voces: «salid aquí judíos, sambenitados, regular, que sino fuese por las órdenes que tienes te habíamos de matar, y voto a Dios que si salieses te mataríamos», todo ello mientras «arremetían a la puerta y daban de golpes en ella muy recios, que al parecer los daban con los pomos de las espadas $\rangle^{87}$.

Junto al recelo que despertaron los descendientes de elementos judaizantes, cabe destacar la animadversión que generaron en sus respectivas localidades ciertos personajes por el mero hecho de ser extranjeros. Dentro de estos personajes cabe incluir también a los representantes de la autoridad, caso de los comisarios que llegaban hasta una localidad a tomar pesquisas sobre ciertos delitos que habían sido denunciados. Así ocurrió, por ejemplo, en Barasoain la noche del 27 de diciembre de 1659, entre las once y doce horas de la noche. Allí se oyó cierto ruido de arcabuces, del que después se supo que fue

83 AGN, Tribunales Reales. Procesos, núm. 12715, fol. 3.

84 AGN, Tribunales Reales. Procesos, núm. 12715, fol. 4.

85 AGN, Tribunales Reales. Procesos, núm. 12715, fol. 4.

86 AGN, Tribunales Reales. Procesos, núm. 12715, fol. 5.

87 AGN, Tribunales Reales. Procesos, núm. 12715, fol. 6. 
herido Juan de Suescun «con un gran perdigón que le hizo sangrar» ${ }^{88}$. Este último, comisario de los Tribunales Reales, fue a recibir cierta información sobre una agresión que se había perpetrado contra Domingo de Garro. Ello provocó esta pesadumbre, pero no sólo quedó ahí ésta, sino que Ambrosio de Alzorriz, Bartolomé de Olagüe y Pedro Mentón, se dedicaron también a cantarle al agredido: «bujarrón, villano, infame, tuerto, cornudo» ${ }^{89}$.

En otros casos los destinatarios fueron personajes que además de ser foráneos habían alcanzado un cargo de enorme relevancia política a escala local. No sorprende, por tanto, que en Tudela el 13 de septiembre de 1740, en torno a las cuatro horas de la mañana, una cuadrilla de unos diez hombres que portaban diferentes instrumentos musicales y armas se dedicasen a cantar distintas pullas. En la plaza en donde vivía Diego José de Olloqui, segundo teniente de alcalde, se dedicaron a lanzar acusaciones contra este personaje. Así, según se recoge, le increparon: "Adiós José Olloqui, venido de Villafranca, ya habéis venido a San Juan y sois alcalde de la vecindad», junto a éstas, otros cantares describían su físico y después de una hora cantándole se retiraron diciéndole: «Adiós José Olloqui, que me voy a acostar, ya estoy cansando por venirte a cantar» ${ }^{90}$, a cuyo tiempo dieron un relincho gritando y se marcharon «cantando a la jota» ${ }^{91}$. Entre sus participantes se encontraron José de Anguas, Diego de León, Sebastián Royo, Felipe Oviedo, Ignacio Madariaga, José de Cuadra, Manuel Gastón, José de Sola, Lorenzo de Garde y José de Aguirre. Parece ser que, detrás de sus ataques se encontró la animadversión que sintieron hacia este personaje por haber venido desde la localidad de Villafranca, a lo que habría que añadir las quejas sobre cómo trató por todos sus medios ser alcalde de Tudela.

Por último, tampoco se libraron de recibir hirientes serenatas ciertos personajes que eran de suma relevancia a escala local. En uno de estos casos fue Antonio Escudero y Muro, caballero de la Orden de San Juan de Jerusalén, quien presentó una queja criminal contra Bernabé García, Valero Virto, José Bardaxi, José Jiménez Bretón, Marcos de Luna, Domingo Virto, Pedro Jiménez, Miguel Delgado y Clemente Salcedo. ¿El motivo? La noche del 22 de agosto de 1714 en Corella, a eso de las doce de la noche, se dedicaron a insultarle tratándole mal de palabra y también le rompieron algunas vidrieras de su casa a base de pedradas. Los acusados «han andado inquietando a dicho barrio con sus guitarras y armados con espadas y broqueles», lo que podría haber motivado «gravísimos inconvenientes ${ }^{22}$. Siendo interrogado sobre lo que

88 AGN, Tribunales Reales. Procesos, núm. 123873, fol. 1.

89 AGN, Tribunales Reales. Procesos, núm. 123873, fol. 2.

90 AGN, Tribunales Reales. Procesos, núm. 126818, fol. 58.

91 AGN, Tribunales Reales. Procesos, núm. 126818, fol. 61.

92 AGN, Tribunales Reales. Procesos, núm. 205341, fol. 1. 
ocurrió aquella noche, y si inquietaron a la gente llevando armas y guitarras, Bernabé García, responde que «en dicha ocasión no inquietaron ni alborotaron dicho barrio y que sólo estuvieron tañendo unas guitarras ${ }^{93}$.

Lo cierto es que en estas situaciones se puede comprobar la importancia que ostentó en este tipo de actuaciones públicas la violencia escrita. Libelos y pasquines pueden ser utilizados como termómetros que son capaces de medir las inquietudes y agitaciones sociales que tuvieron lugar. Es por ello que durante el desarrollo de cualquier acción de naturaleza colectiva su presencia resultó decisiva. Lo más interesante es que gracias a éstos se puede reconstruir el clima de inestabilidad que encierran muchos de estos acontecimientos. Por tanto, no sorprende que en algunas cencerradas fuesen empleados como un instrumento más. Así en 1555 en la localidad navarra de Cascante se hallaron una serie de libelos difamatorios dirigidos contra su alcalde, Sancho Jiménez. Éstos fueron cantados «con gran estruendo y ruido» ${ }^{94}$ a modo de coplas por las calles, y entre los vecinos que se hallaron presentes se encontraron Diego Cunchillos y Miguel Godo. Sin duda, la conflictividad local que existió entre hidalgos y labradores por el control del regimiento se puso de manifiesto a través del contenido de uno de los libelos que fueron encontrados. En éste se refirieron a Sancho Jiménez de la siguiente manera:

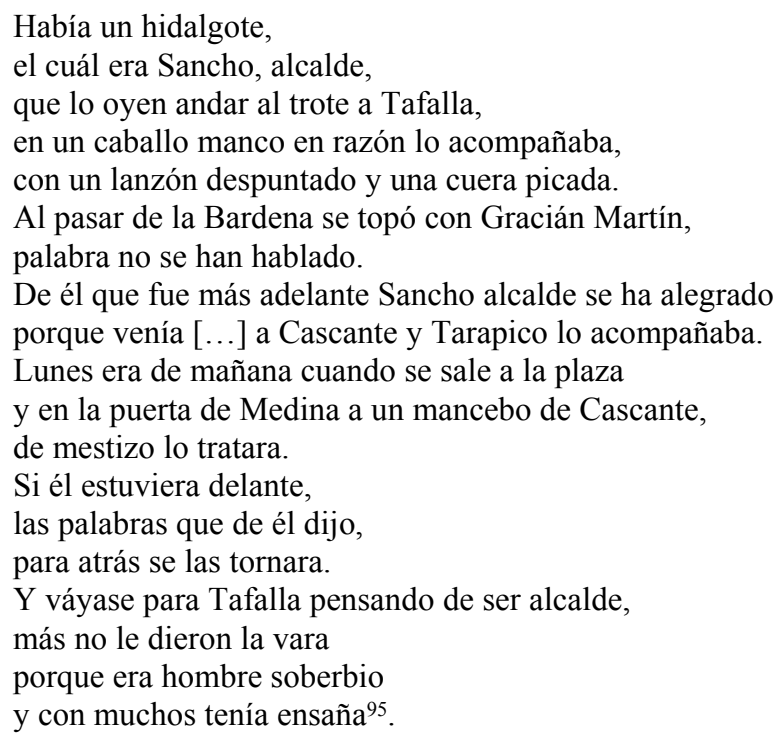

93 AGN, Tribunales Reales. Procesos, núm. 205341, fol. 16.

94 AGN, Tribunales Reales. Procesos, núm. 144725, fol. 2.

95 AGN, Tribunales Reales. Procesos, núm. 144725, fol. 9. 
Tras esta exposición, consideramos que ha quedado puesto de manifiesto que la cencerrada debe ser comprendida como un instrumento popular más allá del mero control de determinados comportamientos considerados como inmorales, sino que debe ser entendida como un mecanismo idóneo para manifestar críticas de calado político, así como también para poner de manifiesto el antagonismo existente entre distintas facciones vecinales. Una vez más ha vuelto a ser resaltada esa idea de la justicia comunitaria que fue ejercida a través de sus denuncias. Sin embargo, el objetivo final de este tipo de matracas fue el control social, a través del cuál manifestar la desaprobación hacia determinados personajes ${ }^{96}$. Lo que sin duda corrobora la hipótesis de Usunáriz $^{97}$ de que la puesta en marcha de estas prácticas culturales trascendió en muchas ocasiones el ámbito doméstico para tratar cuestiones que se caracterizaron por su evidente crítica política. Por tanto, este tipo de cencerradas se erigieron a lo largo de los siglos modernos en una de las formas más legítimas para que el pueblo diese respuesta a sus postulados y expectativas sociopolíticas.

\section{CONCLuSIONES}

Como se ha podido apreciar a través del presente artículo las comunidades vecinales se dedicaron a sancionar a través de condenas populares -como eran las cencerradas o matracas - a todos los vecinos que transgredían las normas establecidas, tratando de poner freno a sus actuaciones para que éstas no afectasen al bienestar del resto de convecinos. Junto a ello, con este tipo de ritos reprobatorios lo que se pone de manifiesto es que el vecindario se interesó en gran medida por una serie de aspectos y de realidades que formaban parte de la vida cotidiana en su ámbito socio-cultural.

Todo ello, sin duda alguna, tiene su origen en el incontestable dominio público sobre los ámbitos privados, puesto que nada escapaba a los inquisitivos ojos de la introspección comunitaria. Por lo que durante la Edad Moderna los rumores se erigieron en componentes básicos de la comunicación popular. Aunque también es cierto que en este juego de fuerzas tuvieron un papel sumamente crucial las autoridades civiles y religiosas al ser bastante permisivas con estas prácticas culturales.

En definitiva, puede concluirse afirmando que tanto cencerradas como matracas se encargaron, como se ha podido constatar en este estudio, de sancionar de un modo colectivo determinadas actuaciones y actitudes que no estaban bien vistas por la sociedad navarra de aquella época. Los numerosos

96 BAZÁN DíAZ, 1995: 271. EIBACH, 2008: 60. MANTECÓN, 2008: 332. MuIR, 1997: 125.

97 USUNÁRIZ GARAYOA, 2006: 254. 
ataques que se centraron en los enlaces matrimoniales, los comportamientos inmorales o las acciones de gobierno no tuvieron otra finalidad que tratar de corregir las desviaciones que se habían originado. En suma, lo que se pretendía a partir de todo acto charivárico era intentar redimir a toda una comunidad, a la vez que se buscaba castigar a quienes habían actuado contra el bienestar local.

\section{BIBLIOGRAFÍA}

Amussen, Susan, "The gendering of popular culture in early modern England", en Tim Harris (ed.), Popular Culture in England, 1500-1850, USA, St. Martin's Press, 1995; 48-68.

Aragonés Subero, Antonio, Danzas, rondas y música popular de Guadalajara, Guadalajara, Patronato de Cultura "Marqués de Santilla", 1986.

Bazán Díaz, Iñaki, Delincuencia y criminalidad en el País Vasco en la transición de la Edad Media a la Moderna, Vitoria-Gasteiz, Gobierno Vasco, 1995.

Bednarski, Steve, "Keeping it in the Family? Domestic violence in the later middle ages: examples from a Provençal town (1340-1403)", en Isabel Davis, Miriam Müller y Sarah Rees (eds.), Love, Marriage and Family Ties in the Later Middle Ages, UK, Brepols, 2003; 277-297.

Beik, William, "The violence of the French crowd from Charivari to Revolution", Past and Present, 197 (Oxford, 2007): 75-110.

Belmont, Nicole, "La fonction symbolique du cortège dans les rituels populaires du mariage", Annales. Histoire, Sciences Sociales, 33/3 (Paris, 1978): 650-655.

Bercé, Yves-Marie, Fête et révolte. Des mentalités populaires du XVIe au XVIIIe siècle, Paris, Hachette, 1976.

Bercé, Yves-Marie, Histoire des Croquants. Étude des soulèvements populaires au XVIIe siècle dans le sud-ouest de la France, Tome I, Genève, Librairie Droz, 1974.

Bonnain-Moerdyk, Roland, "A propos du charivari: discours bourgeois et coutumes populaires”, Annales. Histoire, Sciences Sociales, 32/2 (Paris, 1997): 381-398.

Burguière, Andre, "Réticences théoriques et intégration pratique du remariage dans la France d'Ancien Régime (dix-septième et dix-huitième siècles)", en Jean Dupâquier, Étienne Hélin y Peter Laslett (eds.), Marriage and remarriage in populations of the Past, Londres, Academic Press, 1981; 41-48.

Burguière, Andre, "The Charivari and Religious Repression in France during the Ancien Régime", en Robert Wheaton y Tamara Hareven (eds.), Family and sexuality in French History, Cambridge, Cambridge University Press, 1979; 84-110.

Burguière, Andre, "Le rituel du mariage en France: pratiques ecclésiastiques et pratiques populaires (XVIe-XVIIIe siècle)", Annales. Économies, Sociétés, Civilisations, 33/3 (Paris, 1978): 637-649. 
Candau Chacón, María Luisa, Los delitos y las penas en el mundo eclesiástico sevillano del XVIII, Sevilla, Diputación Provincial de Sevilla, 1993.

Caro Baroja, Julio, "El Charivari en España”, Historia 16, 47 (Madrid, 1980): 54-70.

Caro Baroja, Julio, "Honor y vergüenza", Jean Peristiany (ed.), El concepto del honor en la sociedad mediterránea, Barcelona, Labor, 1968.

Cashmere, John, "The Social Uses of Violence in Ritual: Charivari or Religious Persecution?", European History Quarterly, 21, 3 (Londres, 1991): 291-319.

Castaño Blanco, José Manuel, Conflictividad y violencia. La sociedad sayaguesa en la documentación de los siglos XVI al XIX, Madrid, Instituto de Estudios Zamoranos Florián de Ocampo, 2001.

Chartier, Roger, “Culture populaire et culture politique dans l'Ancien Régime: quelques réflexions", en Keith Baker (ed.), The French Revolution and the creation of Modern Political Culture. Vol I. The political culture of the Old Regime, USA, Pergamon Press, 1986; 243-258.

Davis, Natalie Zemon, Society and Culture in Early Moden France, USA, Stanford University Press, 1975.

Davis, Natalie Zemon, "The reasons of misrule: youth groups and charivaris in sixteenth-century France", Past and Present, 50 (Oxford, 1971): 41-75.

Dean, Trevor, Crime and justice in late medieval Italy, UK, Cambridge University Press, 2007.

Dobash, Robert, "Community response to violence against wives: charivari, abstract justice and patriarchy”, Social Problems, 28/5 (Berkeley, 1981): 563-579.

Eibach, Joachim, "The containment of violence in Central European cities, 15001800", en Robert Mc Mahon (ed.), Crime, Law and Popular Culture in Europe, 1500-1900, UK, Willan Publishing, 2008; 52-73.

Enríquez, José Carlos, "Lo marginal y lo grotesco. Pauta para una caracterización de las exclusiones en las culturas populares y en las hegemonías políticas del Setecientos vizcaíno”, en Iñaki Reguera y César González (eds.), Marginación y exclusión social en el País Vasco, Vitoria-Gasteiz, Universidad del País Vasco, 1999; 211-232.

Enríquez, José Carlos, "La fiesta y sus músicas en el Bilbao del setecientos. Propuestas históricas para el análisis en la cultura urbana de las sensibilidades sonoras", Bidebarrieta, III (Bilbao, 1998): 137-156.

Enríquez, José Carlos, Costumbres festivas y diversiones populares burlescas. Vizcaya, 1700-1833, Bilbao, Beitia, 1996.

Foyster, Edward, Marital Violence. An English Family History, 1660-1857, Cambridge, Cambridge University Press, 2005.

Gauvard, Claude, "Les jeunes à la fin du Moyen Age: une classe d'âge?", en VV.AA. (eds.), Les entrees dans la vie. Initiations et apprentissages, Nancy, Presses Universitaires de Nancy, 1982; 225-244.

Gauvard, Claude y Gokalp, Andre, "Les conduites de bruit et leur signification à la fin du Moyen Age: le Charivari”, Annales. Économies, Sociétés, Civilisations, 29 (Paris, 1974): 693-704. 
Gillis, John, Youth and History. Tradition and Change in European Age Relations, 1770-Present, New York, Academic Press, 1968.

Gómez de Valenzuela, Manuel, "Dos cencerradas en el valle de Tena en el siglo XVIII”, Temas de Antropología Aragonesa, 3 (Huesca, 1987): 118-124.

Greer, Allan, "From folklore to revolution: charivaris and the Lower Canadian rebellion of 1837”, Social History, 15, 1 (Londres, 1990): 25-43.

Grinberg, Martine, "La culture comme enjeu: rituels et pouvoirs (XIVe-XVIIe siècles)", en VV.AA. (eds.), Culture et idéologie dans la genèse de l'État Moderne, Roma, École française de Rome, 1985; 381-392.

Iglesias Estepa, Raquel, "Violencia física y verbal en la Galicia de finales del Antiguo Régimen”, Semata, 19 (Santiago de Compostela, 2008): 135-157.

Ingram, Martin, "Ridings, rough music and mocking rhymes in Early Modern England", en Barry Read (ed.), Popular Culture in Seventeenth-Century England, London, Routledge, 1988; 166-197.

Ingram, Martin, "Ridings, rouge music and the reform of popular culture in early modern England", Past and Present, 105 (Oxford, 1984): 79-113.

Klapisch-Zuber, Christiane, Women, Family, and Ritual in Renaissance Italy, USA, University of Chicago Press, 1985.

Krausman Ben-Amos, Ilana, Adolescence and Youth in Early Modern England, USA, Yale University Press, 1994.

Lebrun, François, La vie conjugale sous l'Ancien Régime, Paris, Armand Colin, 1998.

Lorenzana, Felipe, "Jueces y pleitos. La administración de la justicia en la Baja Extremadura", Hispania, 63/213 (Madrid, 2003): 29-73.

Mantecón, Tomás Antonio, "La violencia en la Castilla urbana del Antiguo Régimen”, en José Ignacio Fortea y Juan Eloy Gelabert (eds.), Ciudades en conflicto (siglos XVI-XVIII), Valladolid, Marcial Pons, 2008; 307-334.

Mantecón, Tomás Antonio, "La violencia marital en la Corona de Castilla durante la Edad Moderna”, en Antonio Irigoyen y Antonio Pérez (eds.), Familia, transmisión y perpetuación (siglos XVI-XIX), Murcia, Universidad de Murcia, 2002; 19-55.

Mantecón, Tomás Antonio, Conflictividad y disciplinamiento social en la Cantabria rural del Antiguo Régimen, Santander, Universidad de Cantabria, 1997.

Marcel-Dubois, Claudie, "Fêtes villageoises et vacarmes cérémoniels ou une musique et son contraire", en Jean Jacquot y Elie Konigson (ed.), Les fêtes de la renaissance, vol. III, Paris, Centre National de la Recherche Scientifique, 1975; 603-615.

Margolin, Jean, "Charivari et mariage ridiculez au temps de la renaissance", en Jean Jacquot y Elie Konigson (ed.), Les fêtes de la renaissance, Vol. III, Paris, Centre National de la Recherche Scientifique, 1975; 579-601.

Medick, Hans, "Village spinning bees: sexual culture and free time among rural Routh in early modern Germany", en Hans Medick y David Warren Sabean (eds.), Interest and Emotion. Essays on the Study of Family and Kinship, Cambridge, Cambridge University Press, 1988; 317-339. 
Mendoza Garrido, Juan Miguel, Delincuencia y represión en la Castilla bajomedieval, Granada, Grupo Editorial Universitario, 1999.

Morgan, George y Rushton, Peter, "Violence, social order, the state and the absence of regulation in working-class communities in eighteenth-century England", en Katherine Watson (ed.), Assaulting the Past. Violence and Civilization in Historical Context, Cambridge, Cambridge University Press, 2007; 104-123.

Muchembled, Roland, Société, cultures et mentalités dans la France moderne, XVIeXVIIIe siècle, Paris, Armand Colin, 1994.

Muchembled, Roland, L'invention de l'homme moderne. Sensibilités, moeurs et comportements collectifs sous l'Ancien Régime, Paris, Fayard, 1988.

Muir, Edward, Ritual in Early Modern Europe, Cambridge, Cambridge University Press, 1997.

Nicolas, Jean, La rébellion française. Mouvements populaires et consciente sociale 1661-1789, Paris, Senil, 2002.

Pellegrin, Nicole, Les bachelleries. Organisations et fêtes de la jeunesse dans le Centre-Ouest, XVe-XVIIIe siècles, Poitiers, Société des Antiquaires de l'Ouest, 1983.

Pérez Teijón, Josefina, "El cortejo en los sainetes y tonadillas del siglo XVIII como transgresión de la moral al uso", en Antonio Romero Ferrer (coord.), VI Encuentro: De la Ilustración al Romanticismo. Juego, fiesta y transgresión, 1750-1850, Cádiz, Universidad de Cádiz, 1995; 137-150.

Pillorget, René, Les mouvements insurrectionnels de Provence entre 1596 et 1715 , Paris, Pedone, 1975.

Pitou, Frédérique, "Jeunesse et désordre social: les coureurs de nuit à Laval au XVIIIe siècle”, Revue Moderne et Contemporaine d'Histoire, 47/1 (Paris, 2000): 69-92.

Pitt-Rivers, Julian, Los hombres de la sierra. Ensayo sociológico sobre un pueblo andaluz, Barcelona, Grijalbo, 1971.

Robisheaux, Thomas, Rural Society and the Search for Order in Early Modern Germany, Cambridge, Cambridge University Press, 1989.

Ruff, Julius, Violence in Early Modern Europe (1500-1800), Cambridge, Cambridge University Press, 2001.

Ruiz Astiz, Javier, La fuerza de la palabra escrita. Amenazas e injurias en la Navarra del Antiguo Régimen, Pamplona, EUNSA, 2012.

Ruiz Astiz, Javier, "Comunidad y cencerrada en el control de la vida matrimonial: Navarra siglos XVI-XVIII”, Memoria y Civilización, 16 (2013). (En prensa).

Ruiz Astiz, Javier, Desórdenes públicos y violencia colectiva en la Navarra moderna (1512-1808), Pamplona, Universidad de Navarra, 2010 (Tesis Doctoral Inédita).

Ruiz Astiz, Javier, "Literatura subversiva: libelos y pasquines en Navarra durante la Edad Moderna", Pliegos Volanderos del GRISO, 12, (Pamplona, 2008): 1-25.

Schindler, Norbert, Rebellion, Community and Custom in Early Modern Germany, Great Britain, Cambridge University Press, 2002. 
Schindler, Norbert, "Los guardianes del desorden. Rituales de la cultura juvenil en los albores de la era moderna", en Giovanni Levi y Jean Claude Schmitt (dirs.), Historia de los jóvenes. I. De la Antigüedad a la Edad Moderna, Madrid, Taurus, 1996; 305-363.

Segalen, Nicole, "Mentalité populaire et remariage en Europe occidentale", en Jean Dupaquier, Étienne Hélin y Peter Laslett (eds.), Marriage and remarriage in populations of the Past, Londres, Academic Press, 1981; 67-77.

Sheridan, Susan, Wife and widow in medieval england, Michigan, University of Michigan Press, 1993.

Shoemaker, Robert, "Male honour and the decline of public violence in eighteenth century London”, Social History, 26/2 (Londres, 2001), 190-208.

Shoemaker, Robert, "The London mob in the early eighteenth century", en Peter Borsay (ed.), The Eighteenth-Century Town. A Reader in English Urban History, 1688-1820, UK, Longman, 1990; 188-222.

Slack, Paul, Rebellion, popular protest and the social order in early moden England, Great Britain, Cambridge University Press, 1984.

Stevenson, John, Popular disturbances in England 1700-1832, Londres, Longman, 1992.

Thompson, Edward Palmer, Costumbres en común, Barcelona, Crítica, 2000.

Thompson, Edward Palmer, "Rough Music: Le Charivari anglais", Annales. Économies, Sociétés, Civilisations, 27/2 (Paris, 1972) : 285-315.

Trinkaus, Charles y Oberman, Heiko (eds.), The pursuit of holiness in late medieval and renaissance religion, Belgium, Brill, 1974.

Underdown, David, Revel, riot and rebellion. Popular Politics and Culture in England, 1603-1660, Great Britain, Oxford University Press, 1985.

Usunáriz Garayoa, Jesús María, "El lenguaje de la cencerrada: burla, violencia y control de la comunidad”, en Rocío García y Jesús María Usunáriz (eds.), Aportaciones a la Historia Social del Lenguaje: España siglos XIV-XVIII, Madrid, Iberoamericana, 2006; 235-260.

Walter, John, "Faces in the crowd: gender and age in the early modern English crowd", en Helen Berry y Elizabeth Foyster (eds.), The Family in Early Modern England, UK, Cambridge University Press, 2008; 96-125.

Recibido: 04-04-2011

Aceptado: 31-07-2012 